\title{
Reversal by L-arginine of a dysfunctional arginine/nitric oxide pathway in the endothelium of the genetic diabetic BB rat
}

\author{
G .M. Pieper, W. Siebeneich, G . M oore-H ilton, A . M . R oza \\ Department of Transplant Surgery, Medical College of Wisconsin, Milwaukee, Wisconsin, USA
}

Summary We examined the effects of acute supplementation with arginine in vitro on endothelium-dependent relaxation in aortic rings taken from female genetic, diabetes-prone BB rats. Sensitivity to norepinephrine-induced contraction was unaltered in rings of diabetic $\mathrm{BB}$ rats compared to rings from non-diabetic littermates. In precontracted rings, acetylcholine produced a concentration-dependent relaxation which was impaired by diabetes. This relaxation was blocked by L-nitroarginine in both control and diabetic rings. Addition of $3 \mathrm{mmol} / \mathrm{l} \mathrm{L}$-arginine (but not D-arginine) enhanced relaxation in diabetic rings similar to that seen in control rings without arginine. $\mathrm{L}$-arginine had no effect on acetylcholine-induced relaxation in control rings. In contrast, relaxation-induced by nitroglycerin in diabetic rings without endothelium was not altered by L-arginine treatment. Thus, a defect in the utilization of arginine by nitric oxide synthase exists in the endothelium of the diabetic BB rat. [Diabetologia (1997) 40: 910-915]

Keywords Nitric oxide, arginine, endothelium, diabetes mellitus.
Recent studies have indicated impaired endotheliumdependent relaxation in arteries of insulin-dependent [1] diabetic (IDDM) patients. The mechanisms for this defect in human blood vessels is not yet understood. Several studies have observed defects in endothelium-dependent relaxation in both conduit and resistance arteries of streptozotocin (STZ)- or alloxaninduced diabetic rats or rabbits (see reviews [2,3]).

Another experimental diabetic model which is not as frequently investigated is the spontaneously diabetic BB (Bio-Breeding) rat despite the fact that this model more closely approximates IDDM in man. Currently, only a limited number of studies regarding

Received: 24 February 1997 and in revised form: 17 April 1997

Corresponding author: Dr. G. M. Pieper, Department of Transplant Surgery, Medical College of Wisconsin, Froedtert Memorial Lutheran Hospital, 9200 West Wisconsin Avenue, Milwaukee, WI 53226, USA

A bbreviations: IDDM, Insulin-dependent diabetes mellitus; $\mathrm{NO}$, nitric oxide; $\mathrm{ACH}$, acetylchloline; NTG, nitroglycerine; L-NA, L-nitroarginine; STZ, streptozotocin; L-ARG, L-arginine. endothelial function have been conducted in this important model [4-7]. More importantly, there is no available information regarding specific defects in nitric oxide (NO) production contributing to defective endothelium-dependent relaxation in this model. In this study, we evaluated the efficacy of supplementation with L-arginine (L-ARG) to improve NO synthase-dependent, endothelium-dependent relaxation in the diabetic $\mathrm{BB}$ rat.

\section{Materials and methods}

Experiments were performed in compliance with the National Institutes of Health "Principles of Laboratory Animal Care" (NIH publication No. 85-23, revised 1985). Progeny of the original $\mathrm{BB}$ rat colony from the University of Pennsylvania were used in breeding pairs to develop a local colony of diabetic and non-diabetic BB rats. Onset of diabetes was regularly monitored using test strips and glucometer and defined by 2 successive days of elevated glucose concentration less than $11 \mathrm{mmol} / \mathrm{l}$. Diabetic animals received s. c. injections of NPH insulin which was varied to maintain a low level of hyperglycaemia (i.e. approximately $12-14 \mathrm{mmol} / \mathrm{l}$ ). Female diabetic BB rats and age-matched, diabetic-resistant littermates were 
housed for 2 months prior to evaluation of endothelial function.

At the termination of the study, animals were anaesthetized with sodium pentobarbital $(65 \mathrm{mg} / \mathrm{kg}$, i.p.). Blood samples were collected from unfasted animals for determination of blood glucose using ExacTech glucometer and test strips (Medisense Inc., Cambridge, Mass., USA.) and for total glycosylated haemoglobin using a commercial diagnostic kit (Sigma Diagnostics, St. Louis, Mo., USA). Descending thoracic aortae were carefully excised and excessive connective tissue and fat were removed. Segments of aorta were cut into rings and mounted on parallel wires in $10 \mathrm{ml}$ isolated Radnoti tissue baths containing Krebs-Henseleit media as previously described [6]. Rings were contracted to an optimal resting tension of $2.0 \mathrm{~g}$ and equilibrated for $1 \mathrm{~h}$. Isometric tension was measured using Radnoti force-displacement transducers and amplifiers connected to a Gould TA6000 recorder.

Concentration-response curves to norepinephrine were performed. After challenge to norepinephrine, each ring was serially washed with fresh medium and equilibrated for 45 min. Rings were then contracted with a submaximal concentration of norepinephrine (e.g. $1 \mu \mathrm{mol} / \mathrm{l})$ which produced approximately $60-65 \%$ maximal response. At the plateau of contraction, concentration-dependent relaxation to acetylcholine $(\mathrm{ACH})$ or nitroglycerin (NTG) was used to evaluate endothelium-dependent compared to endothelium-independent dilation, respectively. In some rings relaxation to $\mathrm{ACH}$ was repeated in rings without intervention or after pretreatment for $20 \mathrm{~min}$ with $100 \mu \mathrm{mol} / \mathrm{l} \mathrm{L}$-nitroarginine (L-NA) or for $45 \mathrm{~min}$ with either $3 \mathrm{mmol} / \mathrm{L}$-ARG or D-ARG. To control for effects of L-ARG specific for vascular smooth muscle, relaxation to NTG was tested in rings without endothelium in the presence compared to the absence of L-ARG.

Statistical analysis. Data (presented as the mean \pm SEM with $\mathrm{n}=$ individual animals) was analysed by analysis of variance followed by Newman Keuls or unpaired t- and paired t-test, where appropriate. A value of $p$ less than 0.05 was set to denote statistical significance.

\section{Results}

A total of 13 diabetic and 11 non-diabetic rat littermates were used for this study. At the conclusion of the study, non-diabetic rats weighed $237 \pm 4 \mathrm{~g}$ while diabetic rats weighed $213 \pm 6 \mathrm{~g}$. Blood glucose concentration was significantly $(p<0.001)$ elevated in diabetic rats $(13.7 \pm 1.7 \mathrm{mmol} / \mathrm{l})$ compared to non-diabetic rats $(3.7 \pm 0.7 \mathrm{mmol} / \mathrm{l})$. Total glycosylated haemoglobin was significantly $(p<0.01)$ elevated (i.e. $4.7 \pm 0.4$ and $2.3 \pm 0.3 \%$, respectively).

There was no significant difference $(p>0.05)$ in either maximal tension development or sensitivity to norepinephrine between non-diabetic and diabetic rings. For example, $\mathrm{pD}_{2}\left(-\log \mathrm{EC}_{50}\right)$ was $6.5 \pm 0.1$ for both groups. Using a submaximal norepinephrine concentration of $1 \mu \mathrm{mol} / 1$ for the relaxation studies, the percentage maximum response was not different between non-diabetic $(67 \pm 4 \%)$ and diabetic $(65 \pm 2 \%)$ rings.

$\mathrm{ACH}$ produced a concentration-dependent relaxation in both non-diabetic and diabetic rings. This

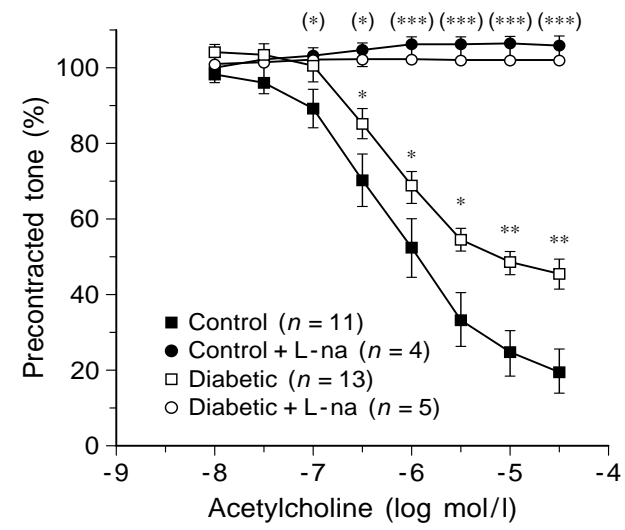

Fig. 1. Endothelium-dependent relaxation to acetylcholine in aortic rings of diabetic BB rat compared to rings from non-diabetic littermates. Also shown is the response in a subset of rings pretreated with $100 \mu \mathrm{mol} / \mathrm{l}$ L-nitroarginine (L-NA). $* p<0.05$ and $* * p<0.01$ vs untreated diabetic and $* p<0.05$ and $* * * p<0.001$ vs corresponding untreated rings

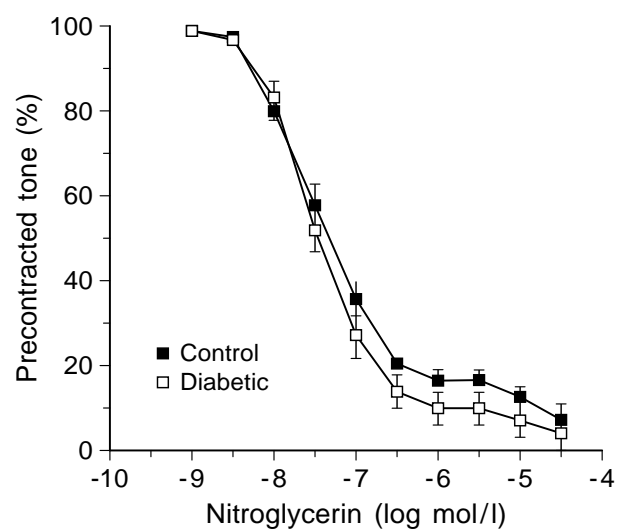

Fig. 2. Endothelium-independent relaxation to nitroglycerin in aortic rings without endothelium from control $\square(n=4)$ and diabetic $\square(n=6)$ BB rats

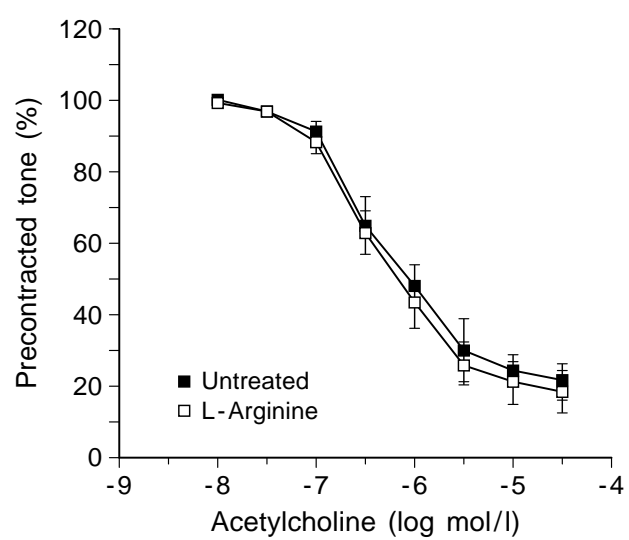

Fig. 3. Relaxation to acetylcholine is not modified by addition of $3 \mathrm{mmol} / \mathrm{l} \mathrm{L}$-arginine $\square$ to control $\square$ rings $(\mathrm{n}=4$ each $)$ 

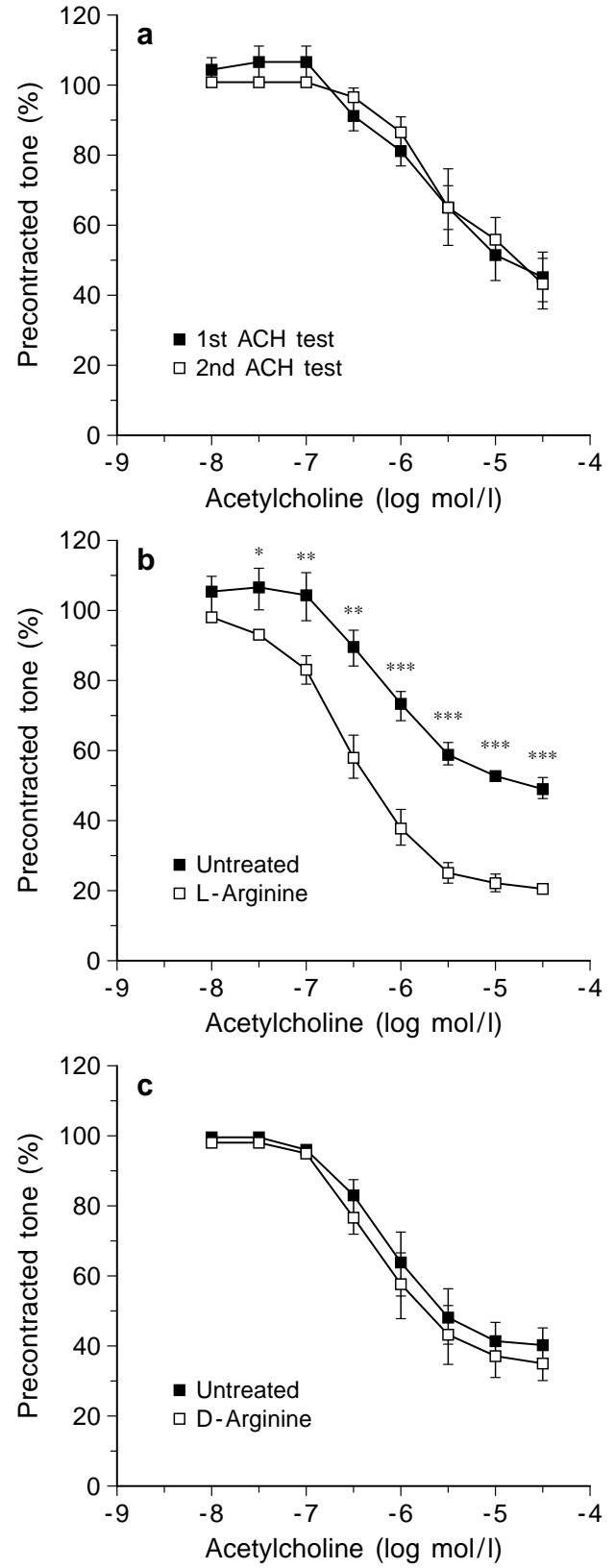

Fig. 4A - C. Relaxation to acetylcholine in diabetic rings before and after incubation with media alone (A), $3 \mathrm{mmol} / \mathrm{l} \mathrm{L}$-arginine (B), or $3 \mathrm{mmol} / \mathrm{l} \mathrm{D}$-arginine (C). Results represent pair-matched data derived from $n=6$ individual diabetic animals. $* p<0.05, * * p<0.01$ and $* * * p<0.001$ vs untreated response

relaxation was blocked by pretreatment with L-NA in both groups. The ACH-induced relaxation was significantly reduced in rings taken from diabetic animals (Fig. 1). In rings without endothelium, NTG-induced relaxation was unaltered by diabetes (Fig. 2).

While L-ARG had no effect on relaxation to $\mathrm{ACH}$ in control rings (Fig. 3), L-ARG enhanced the relaxation to $\mathrm{ACH}$ in diabetic rings (Fig.4). In contrast, $\mathrm{D}-\mathrm{ARG}$ did not alter relaxation to $\mathrm{ACH}$ in diabetic rings. For example maximum relaxation to $\mathrm{ACH}$ was $55 \pm 7$ and $57 \pm 7 \%$ (first and second challenge to

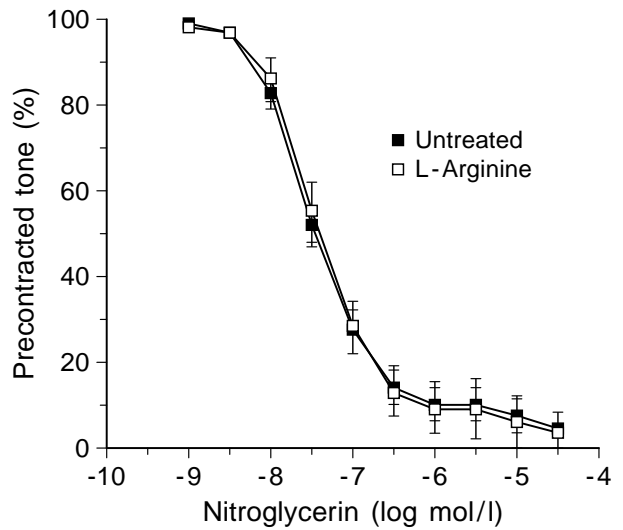

Fig. 5. Addition of $3 \mathrm{mmol} / \mathrm{l} \mathrm{L}$-arginine does not alter the relaxation of diabetic rings without endothelium to the vasodilator, nitroglycerin $(n=6)$

$\mathrm{ACH}$ ), $51 \pm 3$ and $80 \pm 2 \%$ (before and after LARG) and $59 \pm 6$ and $65 \pm 5 \%$ (before and after DARG). Maximum relaxation was $82 \pm 6$ and $79 \pm 5 \%$ in control rings without or with ARG-treatment.

Addition of L-ARG to diabetic rings without endothelium did not modify the relaxation produced by NTG (Fig.5). Pretreatment with L-ARG had no effect on the contractile response to norepinephrine prior to challenge with $\mathrm{ACH}$ (e.g. untreated diabetic $=2.04 \pm 0.13 \mathrm{~g} ;$ L-ARG-treated diabetic $=1.97 \pm$ $0.11 \mathrm{~g})$.

\section{Discussion}

Significant evidence exists for defective endotheliumdependent relaxation in chemically induced diabetic models. In contrast, very little information is available regarding the existence or nature of this dysfunction in the genetic diabetic BB rats. Two early reports $[4,5]$ indicated impaired endothelium-dependent relaxation to $\mathrm{ACH}$ but not to the endothelium-independent vasodilators, sodium nitroprusside or nitrite, in aortic rings of chronically diabetic BB rats. Our results in the present study and elsewhere [6] confirm the impairment of endothelium-dependent but not endothelium-independent relaxation to NTG in this model.

Similarly, a more recent report has demonstrated impaired relaxation to both $\mathrm{ACH}$ and bradykinin in mesenteric resistance vessels in recent-onset, spontaneously diabetic BB/Wistar rats [7]. Taken together this suggests that the phenomenon occurs in both conduit and resistance blood vessels in this model. One potential limitation of all the three other studies using the $\mathrm{BB}$ rat model is that there is no indication whether evaluations were conducted using male or female animals or whether results reflect observations derived from animals of mixed gender. While we have observed that defective endothelium- 
dependent relaxation can be observed in both male and female diabetic BB rats; we have noted that the onset of dysfunction occurs earlier in time in females compared to males (G Pieper, unpublished observations). Thus, we chose female diabetic BB rats and non-diabetic littermates for our study.

Despite the two original studies showing impaired endothelium-dependent relaxation in diabetic $\mathrm{BB}$ rat aorta, no one has yet addressed the specific nature of impaired endothelium-dependent relaxation in conduit arteries of the spontaneously diabetic BB rat. Our study is the first to demonstrate the efficacy of $\mathrm{L}-\mathrm{ARG}$ to reverse defective endothelium-dependent relaxation in aorta of the genetic diabetic BB rat suggesting a defect in the effective utilization of ARG for synthesis of NO by the diabetic endothelium in this model. This effect was demonstrated to be specific for diseased blood vessels in that L-ARG failed to enhance $\mathrm{ACH}$-induced relaxation in aorta taken from non-diabetic littermates. Furthermore, this action is stereospecific in that the D-ARG stereoisomer was ineffective.

It is unlikely that the increased relaxation to $\mathrm{ACH}$ in L-ARG-treated diabetic aorta can be explained by an increased sensitivity of guanylate cyclase activity of diabetic vascular smooth muscle to $\mathrm{NO}$ since NTG-induced relaxation of diabetic aorta without endothelium was not altered. Furthermore, since the contractile response to norepinephrine was not altered in diabetic aorta exposed to L-ARG, this argues against a direct dilator activity of L-ARG contributing to enhanced endothelium-dependent relaxation. Finally, since we previously demonstrated that aminoguanidine, an inhibitor of inducible NO synthase, does not alter responses in aorta of the BB rat [5], the enhanced $\mathrm{ACH}$-induced relaxation of diabetic aorta by L-ARG is unlikely to be explained by providing substrate for $\mathrm{NO}$ by any putative inducible NO synthase present in diabetic vascular smooth muscle.

Our observation showing improvement in endothelium-dependent relaxation of diabetic BB rat aorta by L-ARG is consistent with previous studies conducted in our laboratory in which acute administration of L-ARG in vitro normalized the defective relaxation to $\mathrm{ACH}$ in aorta taken from STZ-induced diabetic Sprague-Dawley and Lewis strain rats $[8,9]$. The observation that L-ARG did not enhance relaxation in aorta of rats of longer diabetes duration [9] suggests that the mechanism of dysfunction might vary with duration of disease. The salient effect of $\mathrm{L}-$ ARG has also been recently shown in coronary arteries of the alloxan-induced diabetic dog evaluated in vivo [10] and in normal mesenteric arteries exposed in vitroto elevated glucose concentrations [11]. Taken together these results suggest that a defect in ARG utilization by endothelial NO synthase for NO production is independent of the model of diabetes.
It has been suggested that endothelial dysfunction in any diabetic model evaluated in vitro reflects the fact that L-ARG is generally absent in the buffer used in organ bath experiments [12]. We find this to be an over simplification for several reasons. First, if endothelial dysfunction were merely due to an absence of ARG in the buffer, one should logically expect diminished relaxation to all endothelium-dependent vasodilators which release NO. This is not the case since the responses to the receptor-independent, endothelium-dependent vasodilator, calcium ionophore, are not altered in aorta of either STZ-induced [8] or genetically induced diabetic BB rats [6]. Secondly, while information in $\mathrm{BB}$ vascular tissue is not available, we have shown that the ARG content in freshly extracted aorta derived from the STZ-induced diabetic rat is already significantly reduced [13]. Thirdly, it is noteworthy that L-ARG can improve function both in vitro and in vivo and that the addition of ARG can improve relaxation in diabetic canine coronaries in vivo despite no significant reduction in plasma ARG concentration. This suggests that diabetes-induced impaired endothelium-dependent relaxation is not likely due merely to an artifact of ex vivo evaluation and that defects in ARG conversion to NO might occur even under conditions of normal extracellular ARG concentration.

Despite the beneficial effects of L-ARG administration given both in vitro and in vivo on endothelium-dependent relaxation in various diabetic models, a recent study revealed that L-ARG did not improve relaxation to $\mathrm{ACH}$ in mesenteric arteries of the spontaneously diabetic BB rat [7]. Since no information was provided regarding insulin dosages, glucose levels and glycosylated haemoglobin in the mesenteric artery study, it is possible that there was tighter glycaemic control compared to our own study. Alternatively, because of variation in experimental design including disease duration, it is not possible to conclude with certainty that the mechanism of endothelial dysfunction differs between conduit and resistance vessels in the spontaneously diabetic BB rat. Accordingly, direct proof for this hypothesis requires a rigorous study of various types of blood vessels conducted in the same laboratory under similar experimental conditions and at similar durations of disease.

One complication when making any comparison between different vascular beds is the observation that L-NA only partially inhibited relaxation to $\mathrm{ACH}$ in mesenteric preparations suggesting that a major component of $\mathrm{ACH}$-induced relaxation in normal mesenteric arteries is not mediated by NO. In contrast, the impaired relaxation to $\mathrm{ACH}$ in mesenteric arteries of newly onset $\mathrm{BB}$ rats and those chronically treated with insulin was completely abolished using L-NA [7]. This observation suggests that the $\mathrm{ACH}$-induced relaxation of mesenteric arteries in these two groups is completely mediated by $\mathrm{NO}$ and 
that diabetes elicits a compensatory increase in the NO-dependent portion of relaxation in this model. In this situation the addition of L-ARG might not be expected to improve relaxation.

The additional observation that indomethacin enhanced relaxation to $\mathrm{ACH}$ in diabetic mesenteric rings suggests that an overlapping increased production of vasoconstrictor prostanoids contributes to endothelial dysfunction. Thus, it is likely that any putative defects which are specific for altered ARG utilization in mesenteric preparations from diabetic $\mathrm{BB}$ rats may be difficult to evaluate due to the masking by other vasoactive factors. While this does not diminish the potential importance of other vasoactive factors contributing to relaxation in this preparation, it considerably complicates any direct analysis of diabetes-induced defects in relaxation which are specific for NO. In contrast, we have shown that $\mathrm{ACH}$-induced relaxation in aorta of both non-diabetic and diabetic $\mathrm{BB}$ rat are likely to be entirely mediated by NO since relaxations were blocked using L-NA and that this relaxation in both groups is insensitive to alteration by indomethacin [6] suggesting that defective $\mathrm{ACH}$-induced relaxation in this model can be explained without the complications of alterations in vasoactive prostanoids.

A more plausible explanation for the divergent observations regarding the efficacy of ARG in the diabetic $\mathrm{BB}$ rat is due to a combination of incubation time and ARG concentration. For example, in the mesenteric artery study [7], blood vessels were exposed for a relatively short period of time (i.e. $20 \mathrm{~min}$ ) compared to the $45 \mathrm{~min}$ duration in our study. In this regard, it has been demonstrated in ARG-deprived bovine aortic endothelial cells incubated with $10 \mu \mathrm{mol} / \mathrm{l}$ extracellular ARG that a minimum of at least $50 \mathrm{~min}$ is required to replete intracellular ARG to steady-state concentrations [14]. The extracellular concentration of ARG used in the mesenteric study was $10 \mu \mathrm{mol} / \mathrm{l}$ (i.e. 300 times lower than that used in our study). This low concentration is nearly 20 times lower than the physiological plasma ARG concentration of approximately $200 \mu \mathrm{mol} / \mathrm{l}$ reported in our laboratory $[8,9,15]$ and elsewhere [16] in different strains of rats and from 7- to 10-times lower than the plasma ARG concentration in STZ-induced diabetic rats. Furthermore, ARG transport into tissue would not be expected to be anywhere near saturation since $10 \mu \mathrm{mol} / \mathrm{l} \mathrm{ARG}$ is approximately 10 times lower than the $\mathrm{K}_{\mathrm{m}}$ for transport by endothelial cells reported to be near $100 \mu \mathrm{mol} / \mathrm{l}$ [17]. Thus, under these experimental conditions, it could reasonably be predicted that the addition of $10 \mu \mathrm{mol} / \mathrm{l}$ ARG might not have any significant impact on endothelial function in these diabetic mesenteric artery preparations. Because of all of these variances, we believe it prudent at this time to caution any extrapolation of the mechanism of endothelial dysfunction in conduit arteries to mechanisms in resistance arteries and vice versa.

The results obtained in the present study using supplementation with L-ARG suggest that deficits in NO production may account for this defect in diabetic $\mathrm{BB}$ rat aorta. This conclusion is consistent with recent studies using coronary microvascular endothelial cells isolated from diabetic BB rats in which decreased basal NO production was observed from intact cells based upon measurements of both nitrate/ nitrite production and ARG-to-citrulline conversion and this could not be accounted for by any intrinsic defects in ARG transporter [18]. In contrast, direct measurement of NO synthase activity in cell homogenates under optimal conditions indicated that there was no intrinsic defect in the NO synthase enzyme in diabetic $\mathrm{BB}$ rat endothelial cells. This suggests that decreased production of NO is specific for intact endothelial cells. This could potentially result from either a limitation in the utilization of the substrate, ARG, either by supply or compartmentalization or limitation in a cofactor for NO synthase, or both, contributing to decreased $\mathrm{NO}$ production by diabetic $\mathrm{BB}$ rat endothelium. Thus, the mechanism of the salient effect of L-ARG on impaired endothelium-dependent relaxation in diabetic blood vessels is likely due to enhanced NO production.

In summary, our experiments provide new information regarding the nature of the defect in endothelium-dependent relaxation in this important experimental model of human IDDM. Our results suggest that a defect exists in efficient utilization of ARG for synthesis of NO by NO synthase in the diabetic $\mathrm{BB}$ rat endothelium and that supplementation with the substrate, ARG, may be one means to reverse endothelial dysfunction.

A cknowledgements. This work was supported by Grant \#HL47072 from the National Institutes of Health, Heart and Lung Institute.

\section{References}

1. Johnstone MT, Creager SJ, Scales KM, Cusco JA, Lee BK, Creager MA (1993) Impaired endothelium-dependent vasodilation in patients with insulin-dependent diabetes mellitus. Circulation 88: 2510-2516

2. Pieper GM, Gross GJ (1991) Endothelial dysfunction in diabetes. In: Rubanyi GM (ed) Cardiovascular significance of endothelium-derived vasoactive factors, Futura Publishing Co., Mount Kisco, New York, pp. 223-249

3. Kamata K, Miyata N, Abiru T, Kasuya T (1992) Functional changes in vascular smooth muscle and endothelium of arteries during diabetes mellitus. Life Sci 50: 1379-1387

4. Meraji S, Jayakody L, Senartne MPJ, Thomson ABR, Kappagoda T (1987) Endothelium-dependent relaxation in aorta of BB rat. Diabetes 36: 978-981

5. Durante W, Sen AK, Sunahara FA (1988) Impairment of endothelium-dependent relaxation in aortae from spontaneously diabetic rats. Br J Pharmacol 94: 463-468 
6. Pieper GM, Moore-Hilton G, Roza AM (1996) Evaluation of the mechanism of endothelial dysfunction in the genetically-diabetic BB rat. Life Sci 58:PL147-PL152

7. Heygate KM, Lawrence IG, Bennett MA, Thurston H (1995) Impaired endothelium-dependent relaxation in isolated resistance arteries of spontaneously diabetic rats. $\mathrm{Br}$ J Pharmacol 116: 3251-3259

8. Pieper GM, Peltier BA (1995) Amelioration by L-arginine of a dysfunctional arginine/nitric oxide pathway in diabetic endothelium. J Cardiovasc Pharmacol 25: 397-403

9. Pieper GM, Jordan M, Adams MB, Roza AM (1995) Syngeneic pancreatic islet transplantation reverses endothelial dysfunction in experimental diabetes. Diabetes 44: 11061113

10. Matsanaga T, Okumura K, Ishizaka H et al. (1996) Impairment of coronary blood flow regulation by endotheliumderived nitric oxide in dogs with alloxan-induced diabetes. J Cardiovasc Pharmacol 28: 60-67

11. Taylor PD, Poston L (1994) The effect of hyperglycaemia on function of rat isolated mesenteric resistance artery. $\mathrm{Br}$ J Pharmacol 113: 801-808

12. Tilton RG, Chang K, Hasan KS et al. (1993) Prevention of diabetic vascular dysfunction by guanidines: inhibition of nitric oxide synthase versus advanced glycation end-product formation. Diabetes 42: 221-232
13. Pieper GM, Dondlinger LA (1996) Extracellular and intracellular arginine deficiency in diabetes leads to reduced endothelium-dependent relaxation and cGMP generation. Circulation 94 [Suppl 1]:I-703 (abstract)

14. Preik-Steinhoff H, Zink S, Rösen P, Kelm M (1995) Transport of L-arginine in arginine-deprived endothelial cells. Biochem Biophys Res Commun 213: 447-453

15. Pieper GM, Siebeneich W, Dondlinger LA (1996) Shortterm oral administration of L-arginine reverses defective endothelium-dependent relaxation and cGMP generation in diabetes. Eur J Pharmacol 317: 317-320

16. Rösen P, Ballhausen T, Stockklauser K (1996) Impairment of endothelium dependent relaxation in the diabetic rat heart: mechanisms and implications. Diab Res Clin Pract 31 [Suppl]:S143-S155

17. Sobrevia L, Cesare P, Yudilevich DL, Mann GE (1995) Diabetes-induced activation of system $\mathrm{y}^{+}$and nitric oxide synthase in human endothelial cells: association with membrane hyperpolarization. J Physiol 489: 183-192

18. Wu G, Meininger CJ (1995) Impaired arginine metabolism and NO synthesis in coronary endothelial cells of the spontaneously diabetic BB rat. Am J Physiol 269 (Heart Circ Physiol 38):H1312-H1318 